\title{
¿Gobernando el cambio? Epistemología neoliberal en el arte de gobernar y en las políticas feministas de la ciudad de Madrid
}

\section{Governing Change? Neoliberal Epistemology in the Art of Governing and the Feminist Policies in the City of Madrid}

CARLOTA CARRETERO GARCÍA (Universidad Complutense de Madrid) y ANDY ERIC CASTILLO PATTON (Universidad Complutense de Madrid)

Artículo recibido: 30 de noviembre de 2018

Solicitud de revisión: 5 de marzo de 2019

Artículo aceptado: 17 de junio de 2019

Carretero García, Carota y Castillo Patton, Andy Eric (2019). ¿Gobernando el cambio? Epistemología neoliberal en el arte de gobernar y en las políticas feministas de la ciudad de Madrid. Recerca. Revista de Pensament i Análisi, 24(2), pp. 39-62.

Resumen

Actualmente, el feminismo, en su condición de movimiento, pero también de teoría viva, fluctuante y sometida a un proceso de revisión, (auto)crítica y disputa permanente, estaría experimentando importantes cambios en el marco de lo que algunas autoras han denominado como cuarta ola. En todo este proceso de emergencia de nuevos feminismos, pero también de pujanza por la categoría y por la significación de la misma, el papel de las políticas e iniciativas públicas - feministas y no feministas- sería de gran relevancia. A lo largo de este trabajo, nos centraremos en analizar algunas propuestas feministas vinculadas a la promoción del empleo y el emprendimiento entre mujeres desarrolladas por el Ayuntamiento de Madrid (2015-2019). De esta manera, analizaremos la producción de sentido y de ideales subjetivos femeninos fomentada en el marco de estas políticas, atendiendo a posibles contradicciones y ambivalencias inherentes a los marcos referenciales movilizados y a su interacción.

Palabras clave: emprendimiento, feminismo, neoliberalismo, subjetividad. 
Abstract

Currently, the feminist social movement and feminist theories are being subjected to a process of resignification, change and conceptual dispute in the frame of what some authors have called the Fourth Wave. This process involves the theoretical development of new feminist approaches and, thus, the struggle for signifying and owning the category feminism. In this complex process, the role of public initiatives and policies is crucial for setting and promoting the hegemonic meaning of this (and any) category. Throughout this paper, we aim to analyse two feminist initiatives developed by Madrid's City Council in order to promote employment from an entrepreneurial approach. By doing so, we will be looking into the production of meaning linked to a feminine ideal subject as well as into a gender biased entrepreneurial discourse.

Key Words: entrepeneurship, feminism, neoliberalism, subjectivity.

\section{INTRODUCCIÓN}

Actualmente, la fortaleza transformadora de los feminismos presenta diferentes expresiones y calado en función del actor y la acción que se contemple. En España, el feminismo se ha articulado en las últimas décadas como una suerte de movimiento pendular que lo ha llevado de la calle a las instituciones, con diferentes resultados según el perfil y el equipo de gobierno en cuestión (Bustelo y Ortbals, 2007). Es en este contexto de formalización efectiva de demandas feministas donde tiene lugar la acción de gobierno del feminismo institucional, un concepto y un enfoque no aceptado por la totalidad del movimiento y de la teoría feminista, en tanto que, desde estas posiciones críticas, se entendería que la profesionalización del feminismo vinculada a su institucionalización conllevaría la anulación de su carácter transformador (Varela, 2008). Con todo, y considerando las diferentes posturas que existen al respecto, a lo largo de este trabajo nos adheriremos a la definición de feminismo institucional conceptualizado como la fórmula por la cual los dispositivos y mecanismos de gobierno incorporan una agenda por la igualdad real entre hombres y mujeres (Reverter, 2011).

Tomando como referencia esta definición, y siguiendo el hilo de análisis desarrollado anteriormente en esta materia, como, por ejemplo, de Celia Valiente $(1998,2006)$, podemos apreciar que el feminismo institucional lleva varias décadas presente en los diferentes gobiernos del Estado español, a todos 
los niveles de la escala territorial, si bien habría estado principalmente vinculado a gobiernos del Partido Socialista Obrero Español (PSOE). En relación con esto, en el análisis que realiza Valiente (1998) de las medidas feministas desarrolladas por parte de los gobiernos municipales de la Comunidad de Madrid (CAM) en la década de 1990, encontramos que los municipios se centrarían, principalmente, en dos pilares, los cuales, a día de hoy, se seguirían manteniendo en la ciudad de Madrid: a) la educación contra las violencias machistas -o violencia contra las mujeres, que sería la terminología usada por dicha auto$\mathrm{ra}-\mathrm{y} b$ ) la promoción del empleo femenino.

A lo largo de este trabajo, nos proponemos analizar varias medidas desarrolladas por el Ayuntamiento de Madrid durante la legislatura 2015-2019 vinculadas a la promoción del empleo y del emprendimiento entre mujeres, concretamente el Premio Emprendedoras y Juntas Emprendemos, si bien también expondremos y analizaremos algunos datos aportados por el informe del Global Entrepreneurship Monitor (GEM) para la ciudad de Madrid. Para el análisis de los a priori y los significados movilizados en el marco de actuación de estas iniciativas, nos serviremos de una interpretación del neoliberalismo entendido como racionalidad de gobierno y como el marco de sentido hegemónico en el que se desarrollarían estas iniciativas —entendiendo la hegemonía como un marco normativo dominante-. En este sentido, el problema del neoliberalismo, siguiendo la propuesta de la teoría foucaultiana

[...] Pasa por saber cómo se puede ajustar el ejercicio global del poder político a los principios de una economía de mercado [...] hasta dónde y en qué medida los principios formales de una economía de mercado podían ser el índice de un arte de gobernar en general (Foucault, 2012: 137).

Este proceso estaría legitimado por el conocimiento experto y estaría dirigido a la administración de las vidas de los sujetos en base a una determinada producción de sentido a la que se le otorgaría la categoría de verdad (Rose, 1996; Oksala, 2013; Prügl, 2015). Por lo tanto, en todo este entramado de producción de sentido promocionado por diversos dispositivos, como empresas, think tanks y diversos productos culturales, las políticas y las iniciativas públicas también jugarían un papel importante (Frade, 2007), incorporando para sí mecanismos ya presentes en la gestión empresarial y promoviendo una gestión de sí en términos empresariales (Rose, 1996; Nicoli y Paltrinieri, 2019).

De este modo, algunas de las preguntas que nos plantearemos irán destinadas a abordar la manera en la que políticas municipales para la promoción 
del empleo entre mujeres, en el contexto del Ayuntamiento de Madrid, podrían estar incorporando a su diseño y producción de sentido y de subjetividades determinadas lógicas y a priori vinculados a la racionalidad neoliberal que, a su vez, podrían interaccionar con ideas feministas concretas. Para ello, nos remitiremos al concepto de neoliberalisation of feminism (Prügl, 2015), así como al de feminismo neoliberal (Rottenberg, 2018a, 2018b) con el propósito de plasmar y problematizar las paradojas y las asunciones vinculadas a la construcción de sentido y de subjetividades por parte de estas políticas. Además, también nos fijaremos en las contradicciones inherentes a la posible integración y promoción de ideas feministas neoliberalizadas (Medina-Vincent, 2018a), así como otros marcos referenciales. De este modo, y en base a lo expuesto hasta este punto, este análisis vendría a plantearse las siguientes preguntas: ¿se adaptan las políticas de empleo mencionadas a los patrones, códigos y expectativas normativas neoliberales? ¿Cuál es el ideal de sujeto mujer que se fomenta desde estas iniciativas? ¿Qué paradojas y contradicciones subyacen a la producción de sentido vinculada a estas iniciativas?

La estructura analítica en torno a la que se articulará esta propuesta desarrolla, en primer lugar, el marco teórico en torno a las ideas de producción subjetiva neoliberal, emprendimiento, género y neoliberalización de ideas feministas. A continuación, en el apartado analítico estudiamos los a priori, contradicciones y paradojas inherentes a la producción de sentido y de subjetividades por parte de las iniciativas Premio Emprendedoras y Juntas Emprendemos centradas en la promoción del empleo entre mujeres en la ciudad de Madrid. Por último, en las conclusiones repasaremos las principales ideas de nuestro estudio, y se presentará una reflexión acerca de las resistencias y tensiones en la labor del gobierno representativo de cara a su interacción con los feminismos movimientistas.

\section{MARCO TEÓRICO: CONSTITUCIÓN DEL EMPRENDIMIENTO Y SU RELACIÓN CON EL GÉNERO}

\subsection{Subjetivación en el régimen del gobierno neoliberal: hacia la empresari- zación de sí}

A lo largo de su análisis genealógico sobre la racionalidad neoliberal, Foucault (2012) contrasta el homo economicus con el empresario de sí, mediante el cual se radicalizaría esta visión androcéntrica y economicista de la sociedad ya 
presente en el homo economicus. En esta línea, desde el liberalismo económico se vincula la naturaleza del individuo con la tendencia al intercambio, entendiéndose como una característica antropológica esencial, mientras que el neoliberalismo introduciría la competencia —en el mercado- como la característica natural y definitoria de los individuos. Si bien esa competencia sería inherente al individuo, debería ser fomentada mediante diversos dispositivos - entre otros, las políticas públicas- para su expresión y, a su vez, protegida de intervenciones estatales o tendencias monopolistas (Reed, 2009).

En esta línea, desde la producción de sentido vinculada a este enfoque mercantilista de la racionalidad neoliberal, se fomentaría que los propios sujetos, con el objetivo de resultar competitivos dentro del mercado, aumentaran constantemente su capital humano mediante la adquisición de competencias que se correspondieran a las demandas del mercado (Reed, 2009; MuñozRodríguez y Santos Ortega, 2017). Es decir, se traspasaría a los individuos la responsabilidad de adaptar sus competencias a las exigencias y necesidades del mercado, fomentando, de esta manera, una suerte de revisión, reinvención e inversión constante en uno mismo (Crespo y Serrano, 2013). Esta producción subjetiva derivada del fomento de la competencia daría lugar, de acuerdo con Rottenberg (2018a, 2018b), a un mundo dividido entre ganadores y perdedores. La responsabilidad individual en la promoción de la inversión constante y reiterada en el aumento del propio capital humano, fruto del fomento de la competencia por parte de instituciones públicas, empresas, think tanks y diversos productos culturales (Frade, 2007) daría lugar a lo que Foucault denomina como sujeto empresario de sí o ideal normativo de emprendedor (Martínez, 2016) como sujeto referencial. De esta manera, se promocionaría una producción subjetiva donde la empresa se erigiría como una sinécdoque de los sujetos, que serían animados a constituirse, no solo como empresarios de sí, sino como marca personal (Rottenberg, 2018a). Asimismo, para promocionar sujetos ambiciosos, motivados, responsables de sí y activos, en oposición a la dependencia y la pasividad, sería necesaria una automonitorización y autointervención continuada, con el objetivo de aspirar a encarnar el ideal normativo de sujeto empresario de sí (Frade, 2007; Serrano, Fernández y Artiaga, 2012; Crespo y Serrano, 2013; Scharff, 2016a). Este énfasis en el gobierno de las subjetividades en términos de intervención individualizada y autorresponsable sería un síntoma de la actual transición de un Estado social a un Estado terapéutico (Illouz, 2007; Serrano, Fernández y Artiaga, 2012; Crespo y Serrano, 2013). En este sentido, el papel de los llamados Estados terapéuticos sería el de promocionar, mediante un discurso psicologicista centrado en la intervención indi- 
vidual y personalizada, la motivación de una correcta actitud y orientación moral hacia el trabajo, donde «la adaptación personal a las nuevas condiciones del mercado de trabajo, [está] jugando un papel central en los procesos de producción de un nuevo tipo de ciudadano» (Crespo y Serrano, 2013: 1112). En otras palabras, se promocionaría la configuración de subjetividades activas, que se hacen cargo de su situación y que, en última instancia, son responsables de las mismas. En este contexto, tal y como explica Ortiz:

[...] las reglas discursivas del nuevo paradigma conectan con las claves del nuevo escenario eco-nómico [sic] y managerial, en el que, junto a la flexibilidad y al fomento de iniciativas individuales, el emprendizaje es el nuevo modelo a seguir [...] (Ortiz, 2018: 232).

De acuerdo con esta autora, la empleabilidad sería la alternativa promocionada desde los poderes públicos a la crisis de la sociedad salarial. Sin embargo, a lo largo de este trabajo, nos referiremos a la promoción de la ética de la empreabilidad como la alternativa que actualmente está siendo fomentada desde instancias públicas tanto como medida de contención del desempleo como forma hegemónica de promoción activa del nuevo empleo.

En toda esta trama de saberes y significados vinculada a la promoción de una subjetividad activa y disciplinada, dicha ética de la empreabilidad se definiría como la clave en la producción de sentido ligada a las demandas políticas de lucha contra el desempleo por parte de los poderes públicos que promulga el emprendimiento. De esta manera, la producción subjetiva se articularía en torno a la promoción del emprendimiento como receta para paliar el desempleo, con la consecuente promoción de un ideal normativo de sujeto que no sería otro que el del emprendedor (Martínez, 2016). Este ideal normativo de emprendedor se constituiría como permanentemente activo, programado para realizarse a sí mismo y preparado para resolver por sí mismo las contradicciones derivadas del régimen laboral y el modelo productivo.

De esta manera, el discurso vinculado a la ética de la empreabilidad trasladaría la responsabilidad de generar empleo a los propios sujetos, mediante la promoción de la iniciativa emprendedora como solución al desempleo. Por ello, el ideal normativo de emprendedor se encontraría permanentemente (auto)sometido a mecanismos de optimización mental de corte terapéutico destinados a la promoción de la autorrealización y del trabajo sobre uno mismo (Ortiz, 2018) mediante una automonitorización y una autorregulación de la psique con vistas a la adhesión a los objetivos de gobierno neoliberal (Han, 2014). No obstante, de acuerdo con Ortiz: 
[...] el énfasis puesto en el desarrollo del espíritu emprendedor obedecería más a la «subjetividad de juicios y creencias» que al producto de una realidad en términos de oportunidades y desarrollo de iniciativas que redunden en el desarrollo social y económico [...] (Ortiz, 2018: 231).

\subsection{Neoliberalismo generizado: genealogía y características del ideal nor- mativo de mujer en el contexto neoliberal}

En todo este entramado de producción subjetiva, vinculado a mecanismos de optimización de corte terapéutico para la promoción de sujetos emprendedores y empresarios de sí, sería pertinente plantearse hasta qué punto existiría diferenciación por género. Con el objetivo de analizar las interacciones entre el género y las lógicas y los a priori neoliberales, este será considerado, en la línea de lo desarrollado por West y Zimmerman (1987), como un proceso constante y reiterado, un doing gender ('hacer género'), que consistiría en «managing situated conduct in the light of normative conceptions of attitudes and activities appropriated for one's sex category» (West y Zimmerman, 1987: 127) que, tal y como explican Pujal y Amigot (2010), sería transversal a cualquier contexto organizacional. Tanto Gherardi (1994) como Butler (2001) ampliarían la dimensión del doing gender de West y Zimmerman y ahondarían en su dimensión psíquica y simbólica, afirmando que es algo que no solo se hace, sino que se piensa y se integra en la subjetividad.

De esta manera, tal y como hemos visto, nos proponemos ahondar en la manera en la que el género y el neoliberalismo interaccionarían en la producción de sentido vinculada a las iniciativas Premio Emprendedoras y Juntas Emprendemos la ciudad de Madrid. A este respecto, el citado trabajo de Valiente (1998) pone de manifiesto que este tipo de iniciativas centradas en la incorporación de las mujeres en el mundo laboral lleva vigente en municipios de la CAM desde principios de 1990, además, contando, en algunos casos, con concejalías o áreas de gobierno propias. No obstante, cabría preguntarse qué subjetividades estaría promocionando este feminismo institucional en la actualidad y en base a qué premisas de partida.

Esta pregunta adquiere mayor pertinencia si contextualizamos el presente análisis en una coyuntura donde las reivindicaciones feministas y, asimismo, el uso de la categoría feminismo habrían vuelto a revalorizarse como parte de lo que Rottenberg (2014: 146) llamaría «momento feminista». En la incorporación de demandas feministas al debate público y a iniciativas de promoción de 
la igualdad de oportunidades, tanto públicas como privadas, Prügl examina cómo:

[...] select feminist movement ideas are being integrated into neoliberal rationalities and logics [...] recognising the diversity and shifting nature of various feminisms and the fluidity of their boundaries [...] (Prügl, 2015: 3).

Esta autora argumenta que se darían «sites of indetermine encounter of feminism and neoliberalism» (Prügl, 2015: 4), por lo que se daría una «neoliberalización de ideas feministas». Tal y como explica Prügl, sería necesario abordar las contradicciones que subyacerían a este fenómeno de integración de ideas feministas en iniciativas privadas o públicas, junto con la interacción con otros marcos referenciales propios, por ejemplo, de profesiones concretas (Scharff, 2016b), así como las maneras en las que se podrían ver transformadas y reformuladas las relaciones de poder. Paralelamente, cabría también fijarse en la producción cultural vinculada a lo que autoras como Rottenberg (2018b) han denominado feminismo neoliberal y en cuyo marco se fomentaría un ideal normativo de balanced women o mujer equilibrada. Este nuevo ideal normativo sería presentado por sus promotoras -entre las que encontraríamos, en un contexto internacional, a Sheryl Sandberg o Ivanka Trump- como nuevo modelo de mujer emancipada y progresista (Rottenberg, 2014, 2018a, 2018b; Medina-Vicent, 2018b) consciente de las desigualdades de género, pero cuyas soluciones son de carácter individual y autorresponsable, generalmente vinculadas a una transformación individual de las propias actitudes y de la autoestima, así como a la dedicación equilibrada y feliz a la familia y a una carrera profesional de éxito.

Este sujeto feminista neoliberal estaría «estrechamente ligado con la lógica del emprendimiento» (Medina-Vicent, 2018b: 5) y apelaría, principalmente, a mujeres occidentales con estudios universitarios y carreras profesionales de éxito. En relación con la promoción de subjetividades emprendedoras o empresarias de sí, estudios como el de Bruni, Gherardi y Poggio (2004) se han ocupado de tratar las coordenadas del subtexto de género inherente a los discursos y las prácticas emprendedoras. De acuerdo con estas autoras, el emprendimiento comprende un subtexto de género vinculado con el dominio simbólico de la masculinidad hegemónica, mientras que el ideal normativo de emprendedor representaría «an acritical reproduction of hegemonic masculinity» (Bruni, Gherardi y Poggio, 2004: 409). De esta manera, según estas autoras, al discurso emprendedor subyacería un orden de género donde los códigos, las expectati- 
vas normativas y los significados vinculados al dominio simbólico de la masculinidad hegemónica se verían exaltados y promocionados y, a la vez, naturalizados y presentados como neutros. Entre esos códigos, características y prescripciones normativas vinculadas a la masculinidad hegemónica se encontrarían la competitividad, la proactividad, la asertividad o el liderazgo (Bruni, Gherardi y Poggio, 2004). Es así que el emprendedor, de acuerdo con estas autoras, personificaría la masculinidad hegemónica, en tanto que, en el marco occidental, se constituiría un imaginario emprendedor, no solo asentado en características y valores vinculados a la masculinidad hegemónica, sino también a cuerpos, imágenes y representaciones ligadas a lo masculino. Paralelamente a este proceso, se daría, de acuerdo con estas autoras, otro proceso de othering the non-male en el que el emprendimiento femenino se pensaría como lo otro. En sintonía con esta argumentación, Oksala (2013: 421) argumenta que «the masculinity of the neoliberal economic subject is usually taken for granted».

Por otro lado, existirían otras investigaciones que vendrían a contradecir parcialmente lo expuesto por Bruni, Gherardi y Poggio (2004). Tal y como explica Scharff (2016a, 2016b), investigaciones más recientes apuntarían a un posicionamiento privilegiado de las mujeres — jóvenes- occidentales como sujetos neoliberales por excelencia. Scharff expone que, en el mundo anglosajón,

[...] public, media and policy discourses have positioned young women as subjects of capacity who can lead responsibilized and self-managed lives through self-application and self-transformation [...] (Scharff, 2016b: 218).

De hecho, Scharff (2016a), en un estudio realizado a partir de más de sesenta entrevistas a mujeres músicas —de clase media-alta y, en su mayoría, blancas - apunta a que sería común que las mujeres configurasen una narración, presentación y percepción de sí mismas en términos empresariales, formulando enunciados referentes a la gestión y la administración de sí en los que la empresa se erigiría como sinécdoque del sujeto. La necesidad de estar activas y dedicadas a la mejora y la optimización constante de sí mismas para adaptarse a las demandas de su entorno laboral estaría muy presente en el discurso de esas mujeres, así como también lo estaría la censura de la vulnerabilidad y el fracaso (Scharff, 2016a). Sin embargo, a pesar de su preminencia, este discurso de empresarialización del yo interaccionaría con otros discursos específicos del contexto laboral de esas mujeres, dando lugar a formulaciones concretas en 
el marco de cada contexto. En base a los resultados de sus entrevistas, Scharff (2016a) concluiría que el sujeto privilegiado de la racionalidad neoliberal sería una mujer joven, de clase media-alta y blanca en tanto que se constituirían como sujetos proclives a la automonitorización y la autooptimización en la gestión de sí en términos empresariales. Si bien estas dos posturas presentarían propuestas que podrían parecer difícilmente conjugables, ambas resultarían de gran utilidad e interés a la hora de pensar y desentrañar las paradojas y las contradicciones del neoliberalismo como proyecto cultural y, más concretamente, del discurso emprendedor.

\section{EL FOMENTO DEL EMPRENDIMIENTO FEMENINO Y LA LUCHA DE MARCOS REFERENCIALES: EL CASO DE LA EXPERIENCIA DE PROGRAMAS EMPRENDEDORES POR PARTE DE AHORA MADRID}

\subsection{Marcos interpretativos y lecturas de género en las iniciativas sobre em- prendimiento en la política municipal de Ahora Madrid}

Gobernar implica poner en práctica decisiones vinculantes a través de los mecanismos institucionales habilitados para tal ejercicio. Por tanto, para hacer efectiva una acción de gobierno, se deben poner en ejecución programas, proyectos o políticas públicas que siempre se sostienen en un discurso. Ahondando en esta cuestión, y de acuerdo con el policy discourse approach que promulgan autores como Hajer (2002), se puede comprender entonces que

[...] los actores estatales, por medio de las políticas públicas, imponen una determinada visión de cómo las instituciones y la sociedad deben entender e intervenir sobre problemas agendados o discutidos, debido a que las políticas públicas se reconocen entonces como hechos discursivos y contenedoras de actos de poder [...] (Jiménez y España, 2013: 98).

Asimismo, autoras como Jónasdóttir y Jones (2008) nos proponen la imprescindible incorporación de una mirada de género que tenga presentes qué marcos interpretativos se formulan y qué relación mantienen con las propuestas feministas las diversas performances gubernamentales tanto en términos prácticos como discursivos. De esta manera, y centrándonos en una acción de gobierno feminista volcada en la promoción de políticas de empleo activo, a lo 
largo de este apartado analizaremos el discurso desplegado en las bases Premio Emprendedoras publicadas en el Boletín Oficial del Ayuntamiento de Madrid (BОАM), así como en el análisis del ecosistema emprendedor de la ciudad de Madrid elaborado por GEM-Madrid (2017), dado su papel de actor experto productor de conocimiento en todo este entramado de producción de sentido. Por último, también ahondaremos en los significados y a priori movilizados en el discurso de la iniciativa Juntas Emprendemos, desarrollada por entidades privadas y del tercer sector en colaboración con el Ayuntamiento de Madrid, concretamente, con los espacios de igualdad de la capital. De esta manera, el análisis se centrará en dos iniciativas basadas en la colaboración públicoprivada, así como en un documento producido por expertos externos al Ayuntamiento, pero por encargo de este.

En primer lugar, en la figura 1 podemos apreciar que la tasa de emprendimiento activo (TEA) de acuerdo con el GEM-Madrid ha experimentado una serie de cambios en su distribución. Por una parte, en lo relativo al emprendimiento femenino, vemos que este ha ido en aumento desde el año 2013, llegando a una tasa bastante próxima a la TEA masculina, tradicionalmente más alta, pero actualmente más inestable según estos datos.

Figura 1

Tasa de emprendimiento activo (TEA) en Madrid (2010-2016)

\begin{tabular}{lcc}
\hline Año & TEA masculina & TEA femenina \\
\hline 2010 & $6,0 \%$ & $3,2 \%$ \\
2011 & $7,2 \%$ & $4,5 \%$ \\
2012 & $7,0 \%$ & $2,7 \%$ \\
2013 & $6,2 \%$ & $3,9 \%$ \\
2014 & $6,3 \%$ & $4,4 \%$ \\
2015 & $7,4 \%$ & $5,7 \%$ \\
2016 & $6,3 \%$ & $5,9 \%$ \\
\hline
\end{tabular}

Fuente: GEM-Madrid (2017: 32)

Por otra parte, según relata el GEM-Madrid, el clásico perfil del emprendedor medio -varón, en torno a los 45 años, con formación en emprendimiento y renta familiar media-alta- entraría en convivencia con el 
surgimiento de un nuevo sujeto emprendedor más joven y diverso, con estudios universitarios, pero con mayores carencias en habilidades emprendedoras (GEM-Madrid, 2017: 34). De este modo, los técnicos del GEM recomiendan la promoción de la cultura del emprendimiento para fomentar

[...] la educación y la formación emprendedora en la escuela, la financiación de las empresas, la potenciación de la educación emprendedora y la reducción de tasas y burocracia [...] (GEM-Madrid, 2017: 7).

Una vez expuestas las cifras y algunas sugerencias del GEM, nos centraremos en la carta de la Delegada de Equidad, Derechos Sociales y Empleo que acompaña al informe de GEM del 2017 para la ciudad de Madrid. La importancia de este documento radicaría en que su presencia en el informe GEM validaría las conclusiones extraídas, así como ciertas verdades, a priori y recetas para paliar el desempleo y promocionar el emprendimiento sugeridas en dicho informe. De esta manera, en el informe GEM-Madrid quedan plasmados, de manera más o menos tácita, los ejes principales en torno a los que se sustenta el paradigma laboral, e ideológico, del emprendimiento y que, podrían estar presentes en mayor o menor medida en las iniciativas que analizaremos a continuación. En base a este informe, desde el Ayuntamiento se propone aceptar la articulación de una serie de medidas que permitan dar respuesta a las demandas de los emprendedores con el objetivo de propiciar las condiciones para sacar adelante y consolidar nuevos negocios en la ciudad de Madrid, tal y como se pone de manifiesto con la siguiente afirmación:

[...] necesitamos herramientas que nos permitan saber las condiciones del entorno para poner en marcha iniciativas empresariales, las capacidades de las personas que quieren llevarlas a cabo, y el potencial de crecimiento y desarrollo de las ya creadas, ya que es este conocimiento el que nos hace adoptar las medidas encaminadas a favorecer la implantación en nuestro territorio, de nuevas y mejores ideas empresariales [...] (Higueras, 2017: 7).

Con este propósito, en la misma carta de presentación que acompaña a este informe, y que en cierta medida se reproduce en otros documentos de convocatoria de concurso público, o de acuerdo de cooperación interinstitucional, se proponen la mejora de

[...] la dinámica del mercado interno, la transferencia de la I+D, las normas sociales y culturales, la educación y la formación emprendedora en la escuela, la financiación de las empresas, la potenciación de la educación emprendedora y la reducción de tasas y burocracia [...] (Higueras, 2017: 7). 
De esta manera, se manifiesta la voluntad por parte del área de Gobierno de Equidad, Derechos Sociales y Empleo de la capital de promocionar tecnologías y estrategias destinadas a promover un cambio de mentalidades dirigido a fomentar el emprendimiento entre la ciudadanía, si bien no se explicitaría qué significados se atribuirían y se movilizarían en relación con lo que es el emprendimiento, cómo se invocaría la atribución de responsabilidades en el marco de este discurso o cómo se entenderían conceptos como iniciativa o autonomía en la propuesta de ideal normativo de sujeto emprendedor. Por otro lado, en este discurso institucional se observan algunas alusiones en las que se explicita el rol de las Administraciones públicas como instancias productoras de sentido y, concretamente, de intervención subjetiva destinada a la promoción de una mentalidad y un hacer emprendedor:

[...] Despertar el espíritu de iniciativa, movilizar a los ciudadanos en acciones orientadas a la actividad productiva y a la creación de la cultura empresarial para el desarrollo económico local, es una tarea que deben asumir las administraciones públicas desde la responsabilidad con los ciudadanos [...] (Higueras, 2017: 7).

En esta línea, la iniciativa del Premio Emprendedoras viene a representar parte de ese apoyo explícito al emprendimiento, concretamente, a las mujeres emprendedoras por parte de la Administración local. Si nos centramos en el análisis del último texto de la convocatoria del premio, 2018, se reconoce el aumento en el número de mujeres emprendedoras que se ha experimentado en los últimos años en la ciudad de Madrid, si bien también se reconoce la desigualdad de oportunidades en la participación de hombres y mujeres en la economía, concretamente, de la actividad emprendedora. Justamente, para tratar de paliar esas desigualdades desde una perspectiva de equilibrio en la participación de este mercado laboral, la Agencia de Desarrollo Económico «Madrid Emprende», perteneciente al área de Gobierno de Equidad, Derechos Sociales y Empleo, lleva impulsando el Premio Emprendedoras - una iniciativa sostenida en colaboración con el suplemento de El Mundo «Yo Dona»desde el año 2011. De acuerdo con el Ayuntamiento de Madrid, el propósito de esta iniciativa es

[...] contribuir al reconocimiento de proyectos originales, creativos e innovadores acometidos por mujeres para promover e impulsar el emprendimiento femenino, así como la generación de empleo e incremento de la actividad económica del país, contribuyendo a eliminar la brecha de género que existe [...] (Ayuntamiento de Madrid, 2018: 5). 
Más adelante, se especifica que, para participar en este concurso, hace falta «disponer de una idea original, creativa e innovadora aplicada a diferentes áreas» (Ayuntamiento de Madrid, 2018: 5) y que, en la valoración de los proyectos, se tendrá en cuenta tanto el grado de innovación como su potencial para promocionar la igualdad de oportunidades entre mujeres. Por lo tanto, estamos hablando de un proyecto dirigido a perfiles con un determinado capital cultural, dada la solicitada predisposición a la creatividad, el ingenio y ciertas dotes manageriales:

[...] Aunque España es un país de personas emprendedoras y de pequeñas y medianas empresas que, en conjunto, representan el $62 \%$ del PIB, la actividad emprendedora suele estar marcada por una participación masculina mayor que la femenina. Las mujeres españolas representan más del $50 \%$ de la población con estudios universitarios, pero en comparación con otros países europeos aún hay pocas mujeres que deciden abrir su propio negocio en España (Ayuntamiento de Madrid, 2018: 4).

De esta manera, podría plantearse la existencia de dos referenciales con los que entender la igualdad de género: uno que alude a la necesidad de facilitar la extensión de actividades emprendedoras entre mujeres desde una perspectiva de igualdad de oportunidades; y otro que remite a una representación vinculada al carácter generizado del emprendimiento y que aboca a las mujeres a adherirse a valores masculinos para salvarse a sí mismas. En este sentido, podría decirse que, si bien se hace una lectura de género respecto a la desigualdad de oportunidades entre hombres y mujeres para sacar adelante una iniciativa emprendedora, no se cuestionan los a priori y significados inherentes y naturalizados en el discurso emprendedor que vincularían los códigos, los valores, las expectativas y las representaciones normativas con el dominio simbólico de la masculinidad hegemónica. Lo paradójico de esta operación de configuración y promoción subjetiva es que, entre las mujeres, se estaría promocionando un discurso que, al carecer de una revisión crítica de la adscripción de género de los códigos, los valores y las expectativas normativas que lo atraviesan, podría implicar que las mujeres a las que va dirigido no aspirasen a obtener el mismo grado de legitimidad como emprendedoras que sus compañeros varones. Es decir, esta estrategia de promoción del emprendimiento femenino fallaría a la hora de realizar una resignificación o reconfiguración de las coordenadas discursivas de género en las que se sitúa a los sujetos emprendedores. No obstante, también podría realizarse otra lectura al respecto y es que, a pesar de la ausencia de una mirada crítica a los a priori de género vinculados al dominio simbólico de la masculinidad hegemónica inherente al dis- 
curso emprendedor, la sola promoción del emprendimiento de mujeres y su cristalización en el aumento de proyectos emprendedores encabezados por mujeres podría contribuir a resignificar esos valores vinculados a la masculinidad hegemónica, dotándolos de nuevos matices y significados que contribuyesen a disolver el nexo semántico y simbólico entre la ética de la empreabilidad y la masculinidad hegemónica.

Asimismo, el Premio Emprendedoras se focaliza en un determinado sujeto de mujer que, por su capital cultural, no solo marca un sesgo de clase o procedencia socioeconómica, sino que también coloca a la mujer emprendedora como elemento distintivo y diferente al sujeto neutro del emprendedor. Esto se aprecia, no tanto en el texto de las convocatorias del premio, sino en el formato de presentación del mismo y del tipo de iniciativas ganadoras, fuertemente vinculadas al dominio simbólico del universo considerado como femenino - organización de eventos sociales y bodas o complementos de moda o ropa interior-. De igual manera, en su función como dispositivo de intervención subjetiva, en tanto que promotor de una mentalidad emprendedora, el Premio Emprendedoras contribuiría a fomentar un cambio de mentalidad dirigido a la promoción del gobierno de uno mismo, la responsabilidad de sí y la autonomía. Esto se observa en no solo la presentación de una «idea madura de negocio que sea original, creativa e innovadora», sino también el compromiso con la administración y una misma que «En caso de ser seleccionadas como ganadoras, deberán constituirse como empresa antes de un plazo no superior a tres meses» (Ayuntamiento de Madrid, 2018: 5).

Esta lógica de activación movilizada por el Premio Emprendedoras no sería un elemento aislado dado que, tal y como hemos visto con anterioridad, la mentalidad emprendedora y la promoción de un ideal normativo de emprendedor se sustentan en una red de actores que lo moldean y canalizan a través de diferentes dispositivos. Si bien el Premio Emprendedoras no deja de ser una iniciativa puntual del sector público en colaboración con el sector privado, su presencia convive y refuerza otros acuerdos de colaboración público-privada, como el programa Juntas Emprendemos. Esta iniciativa, consistente en una red presente en cuatro comunidades autónomas - Madrid, Aragón, Cataluña y País Vasco-, tiene como objeto la «formación y acompañamiento para promover las capacidades emprendedoras de mujeres en situación de vulnerabilidad», poniendo «el acento en fomento del emprendimiento social» (Juntas Emprendemos, 2019). Si bien esta red no es exclusiva de Madrid, vemos que, en el contexto municipal, el Ayuntamiento cede espacios que soportan las actividades de esta red. Esta iniciativa, si bien no opera de manera tan directa 
como el Premio Emprendedoras u otras iniciativas interinstitucionales como el Programa de Emprendimiento Femenino en Sectores Económicos Emergentes y Nuevas Oportunidades de Mercado, contempla la posibilidad de que el emprendimiento se pueda leer con diferentes interpretaciones que interpelan a colectivos más que a individuos.

En el caso de Juntas Emprendemos, vemos que se da una perspectiva mixta donde, sin embargo, se reproducen los esquemas de activación de gobierno de uno mismo, poniendo, sobre todo, el foco en la cuestión de la capacitación y el empoderamiento para emprender. Esta iniciativa resulta de interés para nuestro análisis ya que contempla no solo un programa de promoción del emprendimiento de mujeres más, sino que observa el emprendimiento desde un prisma donde se encuentran una serie de ambivalencias y discursos que ponen en tensión la subjetividad emprendedora que se promulga desde la ética de la empreabilidad. Esto se observa en la declaración de objetivos de este programa piloto, donde se incluyen propósitos como «Potenciar la autoestima, la autonomía y el empoderamiento personal y colectivo de las mujeres participantes para tomar la iniciativa sobre sus vidas», además de proponer

[...] proyectos transformadores y prácticas emprendidas por mujeres, contribuyendo a ampliar referentes habitualmente inexistentes por el estereotipación de la posición hegemónica dominante dentro de la cultura emprendedora [...] (Juntas Emprendemos, 2019).

Asimismo, las tensiones que impulsan esta subjetividad emprendedora conviven con el despliegue de un marco alternativo del emprendimiento que contempla los cuidados en un sentido que afirma ofrecer «espacios "blandos" para el desarrollo de las capacidades emprendedoras que posibilitan que las cosas sucedan» (Juntas Emprendemos, 2019). De este modo, encontramos que el sujeto emprendedor queda marcado por una posición de género donde se activan otros atributos del universo simbólico de lo femenino, donde los cuidados aparecen junto a lo blando o lo amable. Elementos que también podrían vincularse al dominio simbólico de la maternidad.

\section{2 ¿Presencia de un discurso feminista en las acciones de promoción del emprendimiento?}

La ausencia de una crítica al género naturalizado como inherente al discurso de la ética de la empreabilidad y la puesta en práctica de una visión terapéutica de las instituciones, en tanto que promotoras de un enfoque que 
incide en la intervención subjetiva como fórmula para paliar el desempleo, no son los únicos puntos a destacar respecto al discurso del emprendimiento femenino del Premio Emprendedoras. Si bien no se utilizan en ningún momento los términos feminismo o feminista, en las convocatorias del Premio Emprendedoras publicadas en el BOAM, se habla abiertamente de que:

[...] La incorporación de la mujer al ecosistema emprendedor constituye un factor determinante para impulsar la diversidad y la igualdad de géneros, haciendo a las ciudadanas y ciudadanos de un país corresponsables en su desarrollo. El aumento del emprendimiento femenino los últimos años pone de manifiesto que existe una tendencia a romper los modelos masculinos tradicionales [...] todavía hoy existen condicionantes culturales y sociales que llevan a relegar a las mujeres a ciertos trabajos [...] (Ayuntamiento de Madrid, 2018: 4).

Dichos documentos asumen ciertos objetivos, como la igualdad laboral, que, históricamente, han tenido una presencia importante dentro de los feminismos — sobre todo el socialista y el liberal - y que, tal y como vimos en apartados anteriores, constituye un pilar clave dentro del feminismo institucional.

De esta manera, en el caso del Premio Emprendedoras, habría que destacar la integración de ideas feministas vinculadas a la igualdad laboral, fomentada mediante la promoción de una mentalidad emprendedora que haría énfasis en la configuración de sujetos autónomos, responsables, con agencia y capacidad de elección, basándose en la movilización de ideas como la libertad, la independencia o el empoderamiento, atravesadas por connotaciones mercantilistas. La defensa y la promoción de a priori e ideas vinculados al ideal emprendedor, en este caso mujer, en pos de una causa abiertamente autodenominada como feminista y a favor de la igualdad laboral, estaría relacionado con esa neoliberalización de ideas feministas de la que habla Prügl (2015). Esdecir, tendría que ver con la integración de determinadas ideas feministas, como lo son la emancipación, la capacidad de agencia o de elección de las mujeres, a lógicas neoliberales, en este caso, de promoción de un determinado sujeto laboral autorresponsable y dúctil a las demandas del mercado. De este modo, se daría espacio a ideas feministas fácilmente incorporables al marco y a la agenda mercantilista. Esto se observa en el Premio Emprendedoras con la interpelación constante «al reconocimiento de proyectos originales, creativos e innovadores acometidos por mujeres» (Ayuntamiento de Madrid, 2018: 4).

En esta línea, la integración de ideas feministas en el diseño de iniciativas público-privadas, como es el Premio Emprendedoras, atravesadas y condicionadas por lógicas neoliberales, podría contribuir a promover un ideal de sujeto 
mujer cercano al promulgado en el marco del feminismo neoliberal. De este modo, tal y como argumenta Rottenberg (2014), el sujeto mujer promocionado desde el feminismo neoliberal estaría pensado desde una lógica de la (auto)intervención subjetiva basada en operaciones donde el modelo a seguir es el de un sujeto cuya performance se nutre de la competitividad, la autorrealización por medio del trabajo, la autonomía personal o la responsabilidad de sí mismo. En este sentido, nos hallamos ante un paradigma que recicla ciertas premisas del feminismo liberal anglosajón, pero que incorpora otros elementos que, como dice Rottenberg (2014: 164), «helps to forget, yet again, racial inequality by focusing on a post-racial and individualized ("progressive") feminist subject».

De acuerdo con esta lógica, desde el Premio Emprendedoras se tendería a desplegar y promocionar un enfoque universalista y homogeneizante en lo que respecta a la categoría y al sujeto mujeres, al no contemplar otras fuentes de desigualdad social entre las propias mujeres, por ejemplo, nivel socioeconómico, racialización, situación familiar o nivel de estudios. De este modo, el discurso desplegado carecería de un enfoque feminista interseccional (Crenshaw, 1989), en tanto que no se contemplarían otras opresiones además del género en la configuración discursiva del sujeto mujer que promovería esta iniciativa.

Por ello, es interesante observar iniciativas de colaboración privadapública como Juntas Emprendemos, ya que pretenden apelar a un perfil diverso y diferente que trata de encajar un enfoque inclusivo e interseccional. Sin embargo, este contra-perfil también afronta paradojas y ambivalencias debido a una demanda de activación, iniciativa y autorresponsabilidad en sujetos con pocos recursos, tanto en términos económicos como culturales dado que se interpela a «Promover las capacidades emprendedoras de las destinatarias para mejorar su empleabilidad, condiciones sociolaborales y calidad de vida» al mismo tiempo que se «Pone atención a los cuidados en todo el proceso y se ofrecen herramientas para autocuidarse» (Juntas Emprendemos, 2019).

De esta manera, el discurso en torno al emprendimiento promocionado desde esta iniciativa conviviría y se entremezclaría con un marco referencial de promoción de los cuidados y del cuidado de sí desde la perspectiva del feminismo cultural posmoderno como lógica de (auto)gobierno. De este modo, en tanto que categorías políticas, parecería ser que la ética de la empreabilidad y los cuidados representarían los dos marcos referenciales en la promoción del emprendimiento femenino en la iniciativa Juntas Emprendemos. Es así que, si bien se fomentaría la responsabilidad, la autonomía y el empoderamiento económico, estas categorías se encontrarían atravesadas semánticamente por un 
referencial de cuidados desde el cual se buscaría la resignificación de dichas categorías desde el reconocimiento de la interdependencia y el carácter relacional de los sujetos.

Por otro lado, desde Juntas Emprendemos, al ser una iniciativa caracterizada por una intervención continuada, se fomentaría un proceso de constante (auto)transformación, mejora personal y aumento del capital humano con vistas a optimizar la consecución de objetivos, entre los que se encontraría el empoderamiento económico. Estas lógicas, también presentes en el ideal de sujeto mujer promulgado desde el feminismo neoliberal, conllevarían la incorporación de criterios como la productividad y la eficiencia, propios del marco mercantilista, donde la gestión de una misma se da en términos empresariales. De esta manera, desde Juntas Emprendemos se desplegarían marcos referenciales que, por medio de su interacción, contribuirían a una resignificación mutua donde se adivinaría el carácter subyacente de un marco mercantilista en la gestión de sí, a pesar de su crítica a la concepción hegemónica del emprendimiento. Esto se dejaría notar en tanto que aquí el cuidado podría entenderse como una parte más de la buena gestión de una misma, al igual que, si bien al emprendimiento se le darían matices semánticos de interdependencia, tampoco se perdería esa visión de los sujetos como autorresponsables de su propio devenir.

\section{CONCLUSIONES}

Una vez analizados los principales argumentos desplegados en las iniciativas del Premio Emprendedoras y Juntas Emprendemos en Madrid, además de las aportaciones del GEM, se pondría de manifiesto que el marco mercantilista sirve como principal referente para producir verdad y construir sentido en estos programas de empleo destinados a mujeres. No obstante, este marco encontraría una interacción con determinadas ideas feministas vinculadas al empoderamiento económico, la capacidad de elección o la agencia individual, pero también con otros marcos referenciales, como el de los cuidados. Al mismo tiempo, si bien existirían, en algún caso, nuevas representaciones de género del sujeto emprendedor, estas obedecerían a una vinculación con el dominio simbólico de la feminidad hegemónica, al tiempo que otros códigos y expectativas normativas vinculadas a la masculinidad hegemónica no serían cuestionados. 
De este modo, a pesar de que ambas iniciativas estarían atravesadas por lógicas neoliberales e ideas feministas, las manifestaciones semánticas y discursivas serían diferentes en cada iniciativa, sobre todo en relación con el sujeto del emprendimiento al que interpelan. Sin ninguna duda, tal y como se aprecia en las reflexiones de Rottenberg (2018b) y Medina-Vincent (2018a), el Premio Emprendedoras interpela a un tipo de mujer cuyo estatus sería próximo al ideal que propugna el feminismo neoliberal, donde se acomoda un perfil de mujer con un determinado estatus socioeconómico y un determinado capital cultural. Por otra parte, si bien Juntas Emprendemos se focaliza sobre un sujeto emprendedor fuera del imaginario hegemónico, su sujeto de destino tiende a aproximarse a un modelo de empresaria de sí. Por tanto, vemos coincidencias en tanto que se estima que las mujeres emprendedoras parten o aspiran a la autorresponsabilización y autogestión de sus proyectos y proyecciones vitales.

\section{BIBLIOGRAFÍA}

Ayuntamiento de Madrid (2018). Decreto de 23 de marzo de 2018 de la delegada del área de Gobierno de Equidad, Derechos Sociales y Empleo por el que se aprueba la convocatoria pública del «Premio Emprendedoras 2018» del Ayuntamiento de Madrid. Boletín Oficial del Ayuntamiento de Madrid, 8124, 4-13.

Butler, Judith (2001). Mecanismos psíquicos del poder. Teorías sobre la sujeción. Madrid: Cátedra.

Bruni, Attila, Gherardi, Silvia y Poggio, Barbara (2004). Doing Gender, Doing Entrepreneurship: An Ethnographic Account of Intertwined Practices. Gender, Work and Organization, 11(4), 407-429.

Bustelo, María y Ortbals, Candice (2007). The Evolution of Spanish State Feminism: a Fragmented Landscape. En Outshoorn, Joyce y Kantola, Johanna (Eds.). Changing State Feminism (201-223). London: Palgrave Macmillan.

Crespo, Eduardo y Serrano, Amparo (2013). Las paradojas de las políticas de empleo europeas: de la justicia a la terapia. Universitas Psychologica, 12(4), 1111-1124. 
Crenshaw, Kimberlé (1989). Demarginalizing the Intersection of Race and Sex: A Black Feminist Critique of Antidiscrimination Doctrine. Feminist Theory and Antiracist Politics, 140, 139-167.

Foucault, Michel (2012). Nacimiento de la biopolítica. Madrid: Akal.

Frade, Carlos (2007). Gobernar a otros y gobernarse a sí mismo según la razón política liberal. Revista Española de Investigaciones Sociológicas, $119,35^{-64}$.

Gherardi, Silvia (1994). The Gender We Think, the Gender We Do in Our Everyday Organizational Lives. Human Relations, 47 (6), 591609.

Global Entrepreneurship Monitor-Madrid (2017). Actividad emprendedora en la ciudad de Madrid. Informe Global Entrepreneurship Monitor 2016. Madrid: Ayuntamiento de Madrid / Centro de Iniciativas Emprendedoras (CIDADE) de la Universidad Autónoma de Madrid.

Hajer, Maarten (2002). Discourse Analysis and the Study of Policy Making. European Political Science, 2(1), 61-65.

Han, Byung-Chul (2014). Psicopolítica. Barcelona: Herder Editorial.

Higueras, Marta (2017). Carta de presentación. GEM-Madrid, Actividad emprendedora en la ciudad de Madrid. Informe Global Entrepreneurship Monitor 2016. Madrid: Ayuntamiento de Madrid/Centro de Iniciativas Emprendedoras (CIDADE) de la Universidad Autónoma de Madrid.

Illouz, Eva (2007). Cold Intimacies: The Making of Emotional Capitalism. Cambridge: Polity Press.

Jiménez, Hernán David y España, Milena (2013). Enfoque deliberativo para el análisis de las políticas públicas. Una perspectiva de Maarten Hajer. FORUM, 1(4), 95-112.

Jónasdóttir, Anna G. y Jones, Kathleen B. (2008). The political interests of gender revisited: reconstructing feminist theory and political research. En Jónasdóttir, Anna y Jones, Kathleen (Eds.). The political interests of gender revisited. Redoing theory and research with a feminist face (1-16). Manchester: Manchester University Press.

Juntas Emprendemos (2019). Juntas Emprendemos. Promoción y visibilización de proyectos emprendedores cooperativos de mujeres. Recuperado de:

http://www.juntasemprendemos.net/es/category/edicion/edicion2018/ [Consultado el 25 de noviembre de 2018]. 
Martínez, Laureano (2016). El discurso europeo sobre el emprendimiento. Políticas públicas, trabajo y subjetividad en el marco de la gobernanza económica europea. Tesis doctoral. Universidad Pública de Navarra.

Medina-Vicent, María (2018a). Feminisme neoliberal: un oxímoron? Quaderns de Filosofía, 2, 75-101.

Medina-Vicent, María (2018b). Mujeres y emprendimiento a través de Lean In: una perspectiva crítica. Cuadernos de Relaciones Laborales, $36(2), 305-323$.

Muñoz-Rodríguez, David y Santos Ortega, Antonio (2017). Las cárceles del capital humano: trabajo y vidas precarias en la juventud universitaria. Recerca. Revista de Pensament i Análisi, 20, 59-78.

Nicoli, Massimiliano y Paltrinieri, Luca (2019). «It's still day one». El tránsito del empresario de sí mismo a la start-up existencial en el marco de las transformaciones de la racionalidad neoliberal. Recerca. Revista de Pensament i Análisi, 24(1), 37-60.

Oksala, Johanna (2013). Feminism and Neoliberal Governmentality. Foucault Studies, 16, 32-53.

Ortiz, Pilar (2018). La construcción del discurso político del emprendimiento en España. Realidad e ideología. PAPERS, 103(2), 229-253.

Prügl, Elisabeth (2015). Neoliberalising Feminism. New Political Economy, 20(4), 614-631.

Pujal, Margot y Amigot, Patricia (2010). El binarismo de género como dispositivo de poder social, corporal y subjetivo. Quaderns de Psicologia, 12(2), 131-148.

Reed, Jason. (2009). A genealogy of homo-economicus: neoliberalism and the production of subjectivity. Foucault Studies, 6, 25-36.

Reverter, Sonia (2011). Los retos del feminismo institucional. Daimon. Revista Internacional de Filosofía, 4, 223-229.

Rose, Nikolas (1996). Governing advanced liberal democracies. En Barry, Andrew, Osborne, Thomas y Rose, Nikolas (Eds.). Foucault and Political Reason: Liberalism, Neo-liberalism, and Rationalities of Government (37-64). Chicago: The University of Chicago Press.

Rottenberg, Catherine (2014). Happiness and the Liberal Imagination: How Superwoman Became Balanced. Feminist Studies, 40(1), 144168.

Rottenberg, Catherine (2018a). Women who work: the limits of the neoliberal feminist paradigm. Gender, Work \& Organization, 1-10. 
Rottenberg, Catherine (2018b). The Rise of Neoliberal Feminism. New York: Oxford University Press.

Scharff, Christina (2016a). The Psychic Life of Neoliberalism: Mapping the Contours of Entrepreneurial Subjectivity. Theory, Culture \& Society, 33(6), 107-122.

Scharff, Christina (2016b). Gender and Neoliberalism. Young Women as Ideal Neoliberal Subjects. En Springer, Simon, Birch, Kean y MacLeavy, Julie (Eds.). The Handbook of Neoliberalism (217-226). New York: Routledge.

Serrano, Amparo, Fernández, Carlos J. y Artiaga, Alba (2012). Ingenierías de la subjetividad: el caso de la orientación para el desempleo. Revista Española de Investigaciones Sociológicas, 138, 41-62.

Valiente, Celia (1998). Feminismo de Estado en los ayuntamientos de la Comunidad Autónoma de Madrid. Gestión y análisis de políticas públicas, 14, 173-190.

Valiente, Celia (2006). El feminismo de Estado en España: el Instituto de la Mujer (1983-2003). Valencia: Institut Universitari d'Estudis de la Dona.

Varela, Nuria (2008). Feminismo para principiantes. Buenos Aires: B De Bolsillo.

West, Candance y Zimmerman, Don H. (1987). Doing Gender. Gender and Society, $1(2), 125^{-151 .}$ 
62 RECERCA · DOI: http://dx.doi.org/10.6035/Recerca.2019.24.2.3 - ISSN: 1130-6149-pp. 39-62 